\title{
Interdisciplinary management of multidrug-resistant tuberculosis in an HIV-infected patient: case report
}

\author{
Bogusz J. Aksak-Wąs ${ }^{1}$, Magdalena Leszczyszyn-Pynka ${ }^{1}$, Miłosz Parczewski ${ }^{1}$, Adam Krzyształowski ${ }^{2}$ \\ ${ }^{1}$ Department of Infectious, Tropical Diseases and Immune Deficiency, Pomeranian Medical University in Szczecin, Poland \\ ${ }^{2}$ Department of Radiology, Regional Hospital, Szczecin, Poland
}

\begin{abstract}
Although the incidence of new tuberculosis (TB) cases in the general population is decreasing every year, the frequency of multi-drug-resistant TB is rising, especially throughout Eastern Europe, with the risk of further spread to other European counties. Nowadays, in the era of easy international travel, individualised exposure and drug response patterns need to be reviewed. Even in countries with small numbers of resistant Mycobacteria cases, MDR or XDR-TB should be considered, especially when there is a poor response to antituberculotic therapy.

In the clinical practice, it is often necessary to include a surgical approach, with the excision of infectious tuberculomatous foci, supplementing tuberculostatic treatment. Treatment success is commonly dependent on such a combined approach. Such multidisciplinary care remains a significant and multifaceted problem, especially in poorer countries.

In this paper, we present the case of a 46-year-old HIV-infected patient diagnosed with multidrugresistant, multiorgan TB. The patient, of Polish nationality, previously working in Norway, presented in 2011 as newly diagnosed HIV-1 infection with a suspicion of TB. On admission, the patient's condition was severe with clinical features of wasting and symptoms of pulmonary and extrapulmonary TB infection. Standard treatment outcome was poor, with clinical response achieved only after confirmation of multidrug-resistant TB and optimisation of the therapy. Tuberculostatic treatment was supplemented with multiple surgical procedures, aimed at the diagnostics and allowing treatment of infectious foci. Therapy required complex management of two primary infections (HIV and TB) and multiple adverse-effects and coinfections that occurred during follow-up.
\end{abstract}

HIV AIDS Rev 2017; 16, 3: 198-203

DOI: https://doi.org/10.5114/hivar.2017.70947

Key words: tuberculosis, HIV, AIDS, multidrug-resistant, extensively drug-resistant tuberculosis.

\section{Introduction}

Mycobacterium tuberculosis was discovered in 1882 by the German scientist Robert Koch [1]. Historically, tuberculosis (TB) was treated in an operative manner: thoracoplasty and ball plombage was used to collapse the underlying lung and induce pneumothorax [2]. The first surgeries included pulmonary resection and pneumonectomy or pleuropneumonectomy [2]. It took almost 40 years to discover the first anti-infectious agent for this pathogen. In 1921, for the first time, streptomycin and 4-aminosalycic acid were used for the treatment of the disease. As a result, the role of surgi-
Address for correspondence: Bogusz J. Aksak-Wąs, Department of Infectious, Tropical Diseases and Immune Deficiency, Pomeranian Medical University in Szczecin, 6/1 Jarowita St., 70-501 Szczecin, Poland, e-mail: bogusz.aw@gmail.com
Article history:

Received: 29.05.2017

Received in revised form: 02.07.2017

Accepted: 04.10.2017

Available online: 13.10 .2017
International Journal of HIV-Related Problems

HIV \& AIDS

R e vi e w 
cal treatment was diminished. Modern treatment consists of fixed combinations of tuberculostatic agents taken for six months or more. For patients with no history of TB or no information on the mycobacterial drug resistance, two months of rifampicin, isoniaside, ethambutol, and pyrazinamide should be introduced and followed by consolidation with four months of rifampicin combined with isoniaside. Certainly, treatment should be optimised based on the history of mycobacterial resistance, especially with infection possibly acquired from a patient with previously reported drug resistance amongst mycobacteria. Treatment should be prolonged when new foci of TB are found (for example abscesses), or when there is no mycobacterial response to the treatment (it is not possible to obtain negative sputum results for acid-fast bacilli) [3].

In 20128.6 million people developed TB worldwide, of these 1.1 million (about 13\%) were also HIV-positive [4]. Tuberculosis in HIV-positive patients remains a significant problem, firstly because diagnosis of TB is regarded as opportunistic infection and acquired immunodeficiency syndrome (AIDS)-defining condition, but also because of the fact that due to poor cellular immunity among patients with HIV, treatment of TB is often complicated and commonly needs to be prolonged. Additionally, it requires cautious assessment of drug-drug interactions between common four-drug treatment of TB and three-drug antiretroviral treatment. Introduction of ART and tuberculostatic treatment is a priority, followed by co-trimoxazole treatment, which substantially reduces mortality in HIV-positive TB patients $[5,6]$. In susceptible cases, it is not necessary to alter any of the standard anti-TB treatment, although the option of taking drugs not daily but a couple of times per week is no longer recommended [3].

Nowadays, as MDR-TB (multidrug-resistant TB, resistance to at least isoniazid and rifampicin) and XDR-TB (extensively drug-resistant $\mathrm{TB}$, with resistance to at least isoniazid, rifampicin, and at least one of the injectable drugs such as kanamycin, amikacin, capreomycin, or streptomycin) is evolving and increasing its prevalence in Europe and worldwide, surgical intervention is re-considered as an integrated part of patient therapy, not only in MDR-TB cases, but in regular TB cases, especially when multiple organs and lymph nodes are involved [7].

The WHO reported 450,000 of new MDR-TB cases in 2012, and 170,000 deaths caused by multidrug-resistant TB [7]. Unfortunately, the connection between HIV and MDR-TB is not obvious. Treatment of MDR and XDR-TB is based on mycobacterial susceptibility to drugs; it should consist of four drugs with either certain, or almost certain, efficacy. The WHO defined five groups of drugs for treatment choices, one should choose in hierarchical order from one to five, with the first being the most effective to the fifth with an unclear role in the treatment of drug-resistant TB. The first group consists of the classic, first-line tuberculostatics such as pyrazinamide, ethambutol, rifampicin/rifabutin and isoniazid. The second includes injectable agents mostly from the aminoglycoside group, such as kanamycin, amikacin, capreomycin, and streptomycin. In the third group fluoroquinolones are listed: levofloxacin, moxifloxacin, ofloxacin, and gatifloxacin. The fourth group consists of oral bacteriostatic agents of the second line: para-aminosalicylic acid, cycloserine, terizidone, ethionamide, and prothionamide. Role of the fifth group of agents is the least clear in the treatment MDR-TB, with drugs such as: clofazimine, linezolid, amoxicillin/clavulanate, thioacetazone, imipenem/cilastatin, clarithromycin, and isoniaside in high dosage, or bedaquiline [8].

\section{Case report}

In May 2011 a male Caucasian patient aged 43 years, of Polish nationality, was referred to the Department of Infectious, Tropical Diseases and Immune Deficiency in Szczecin, Poland from the Department of Lung Diseases and Tuberculosis, Szczecin, Poland because of general severe clinical condition, suspected CMV-retinitis, newly diagnosed multiorgan $\mathrm{TB}$, deteriorating kidney function as well as HIV infection, which was thought to be newly diagnosed.

On medical examination, it was revealed that the patient was also complaining of persistent cough (chest X-ray was taken - Figure 1) and weight loss (BMI $19 \mathrm{~kg} / \mathrm{m}^{2}$ ) developing in the last several months. On review of the case history it was surprisingly found that this patient was initially diagnosed with heterosexually transmitted HIV-1 in the year 1992 and was lost to follow-up in 1996; all data on the previous diagnosis were not disclosed by the patient on admittance to the Infectious Diseases Department or to the Department of Lung Diseases and Tuberculosis. We had no data about treatment of HIV infection in 1992; however, the patient had previously been admitted to the Department of Infectious Diseases in Szczecin in 1996 for short-term diagnostics. During this early infection period the patient was asymptomatic, and in accordance to the 1996 antiretroviral treatment guidelines, antiretroviral therapy was deferred. At that time, patient was HbsAg negative, anti-HCV negative, anti-CMV IgG negative, tuberculin skin test proved negative, laboratory markers of the liver and kidney function within the normal range. Lymphocyte CD4 count was 653 cells $/ \mathrm{mm}^{3}$, HIV RNA was not measured, in the fundus of the eye no abnormalities were detected. Dental caries required urgent dental treatment. Additionally, a history of Neisseria gonorrhoea infection as well as past acute hepatitis B with elimination of the HBs antigen was disclosed. Employment history: the patient had been working in Norway from 2003 to 2011 (a total of eight years) as a physical worker in the shipyard (he was a hull assembler), in close contact with citizens of Russia and Lithuania. At the time of diagnosis of TBC he was married and had fathered four children aged 12, 7, 5, and 3 years, who all proved HIV-negative and did not present with any symptoms or laboratory signs of TB. The patient had received a BCG vaccination as an infant.

Clinically, on admittance in 2011, the patient's condition was unstable, with remittent fever (maximum values of $39-40^{\circ} \mathrm{C}$ ), despite standard TB treatment (rifampicin, etham- 
butol, isoniazid, pyrazinamide, which had been introduced by the Department of Lung Diseases and Tuberculosis in April 2011), deteriorating kidney function (creatinine level at first $2.26 \mathrm{mg} / \mathrm{dl}$ than up to $3.15 \mathrm{mg} / \mathrm{dl}$; eGFR $25 \mathrm{ml} /$ $\mathrm{min} / 1.73 \mathrm{~m}^{2}$ ), anaemia with $\mathrm{Hb} 6.7 \mathrm{~g} / \mathrm{dl}$ and erythrocyte levels of $2.45 \mathrm{mln} / \mathrm{mm}^{3}$, and bilirubin level on admittance $7.15 \mathrm{mg} / \mathrm{dl}$ with subsequent increase to the maximal value of $18.45 \mathrm{mg} / \mathrm{dl}$. Additionally, inflammatory parameters were notably increased - procalcitonin up to $9.12 \mathrm{ng} / \mathrm{ml}$ and initial CRP of $268.7 \mathrm{mg} / \mathrm{l}$. Hypoalbuminaemia was deep, with an albumin level of $1.44 \mathrm{~g} / \mathrm{dl}$. In the course of in-hospital treatment, cultures from the oral cavity disclosed multiple thrush infections Candida krusei, C. albicans, C. glabrata, and C. inconspicua were cultured. Stool, blood, and urine culture were repeatedly negative for pathogens (stool and urine twice, blood cultures negative nine times). Moreover, on admittance sputum returned three-times acid-fast bacilli positive with confirmation in positive liquid culture (obtained in June 2011). TBC-PCR test was not performed as it was not available at the time.

Baseline lymphocyte CD4 count was 37 cells $/ \mathrm{mm}^{3}$, CD8 - 353 cells $/ \mathrm{mm}^{3}$, CD4/CD8 ratio of $0.11, \mathrm{HIV}-1$ was subtype B, with no primary transmitted resistance. HIV RNA 61 copies $/ \mathrm{ml}$. The patient was placed on antiretroviral treatment while still in the Department of Lung Diseases and Tuberculosis. Initial treatment was with tenofovir disoproxil/ emticritabine (Truvada 1 tablet qd) combined with efavirenz (Stocrin) $800 \mathrm{mg} / \mathrm{qd}$.

On admission to the Infectious Diseases Department, no significant CMV replication was noted (CMV DNA $<1000$ copies/ml) with CMV-IgG positive, CMV-IgM negative; as expected the patient was anti-HBc total - positive. Initial ophthalmologic consultation suggested possibility of CMV-retinitis - in retinas of both right and left eyes, below optic nerve disc singular grey foci with sharp edges were noted. For this reason, intravenous ganciclovir (250 mg bid, 21 days) was initiated; oral co-trimoxazole (960 mg every other day) for primary prophylaxis of Pneumocystis jirovecii infection was also included to the treatment.

During hospital stay additional tests were performed: in abdominal ultrasound scan (Figure 2) multiple small hypoechogenic foci in liver, up to $0.9 \mathrm{~cm}$, were found. The spleen was enlarged to $14.5 \mathrm{~cm}$, also with various hypoechogenic foci with diameter up to $0.7 \mathrm{~cm}$.

In the initial days of the in-hospital stay the patient developed ileus, treated without surgical intervention. Because of multiorgan TB and CMV-retinitis AIDS was diagnosed. The patient was continued on the first-line antituberculotic treatment: rifampicin, isoniazid, ethambutol, pyrazinamide without intramuscular streptomycin at first, and when a creatine level of $1.35 \mathrm{mg} / \mathrm{dl}$ was achieved, streptomycin was introduced (1.0 gram/day intramuscularly). For candidiasis patient was initially treated with oral fluconazole $(100 \mathrm{mg}$ bid) and subsequently, due to lack of improvement and selection of resistant candida spp., with oral voriconazole (at first day $0.4 \mathrm{~g}$ twice a day then $0.2 \mathrm{~g}$ twice a day).

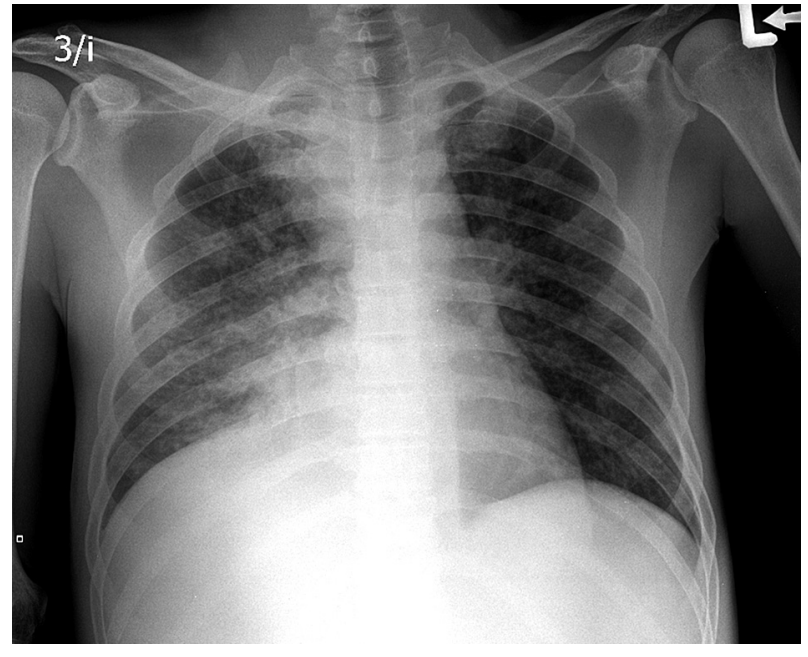

Figure 1. Chest $\mathrm{X}$-ray at the beginning of the diagnostic process

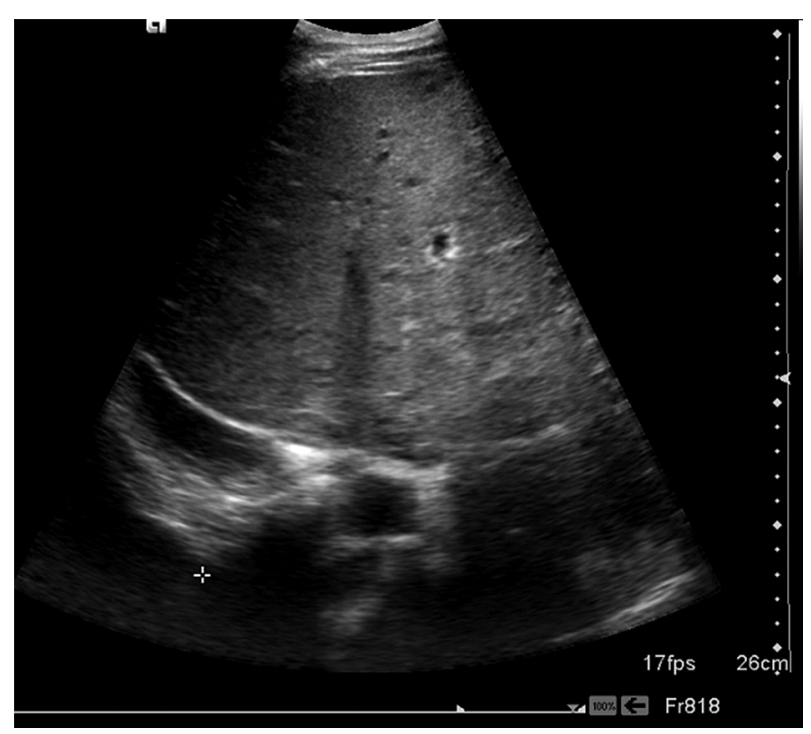

Figure 2. Abdominal ultrasound scan: multiple hypoechogenic foci in the liver

Due to exacerbating anaemia ( $\mathrm{Hb} 6.7 \mathrm{~g} / \mathrm{dl}$; erythrocytes $2.45 \mathrm{mln}$ ) repeated erythrocyte mass transfusions were required; in total eight units were transfused in two months, May and June. Albumin transfusions were also performed. After further increase of bilirubin levels up to $18.45 \mathrm{mg} / \mathrm{dl}$ during the first month rifampicin and isoniaside were excluded from treatment.

In the fourth week of hospitalisation, in the right supraclavicular region, a tumour appeared, which was punctured - purulent contents were found, with neither aerobic, anaerobic bacteria, nor fungi found; however, AFB cultures proved positive.

In magnetic resonance of the head (Figure 3 and 4 ) a couple of foci that were contrast enhancing in circumferential regions of the brain, with oedema around changes, were 


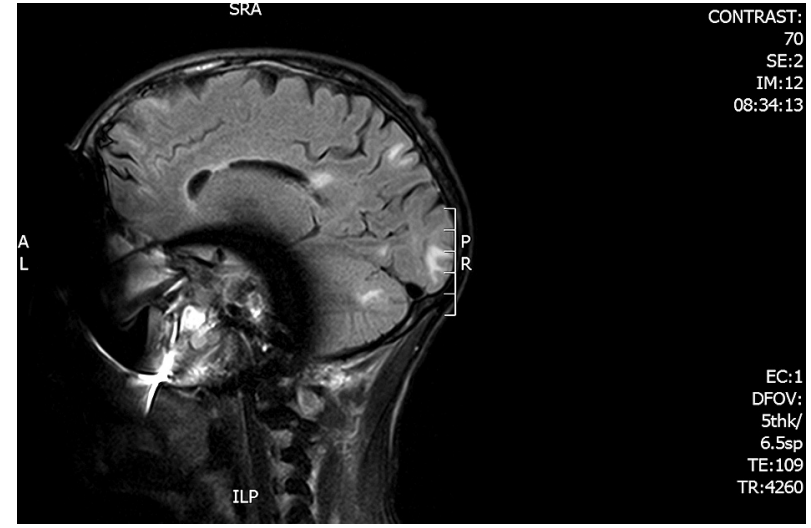

Figure 3. Magnetic resonance of the head: multiple contrast enhancing foci

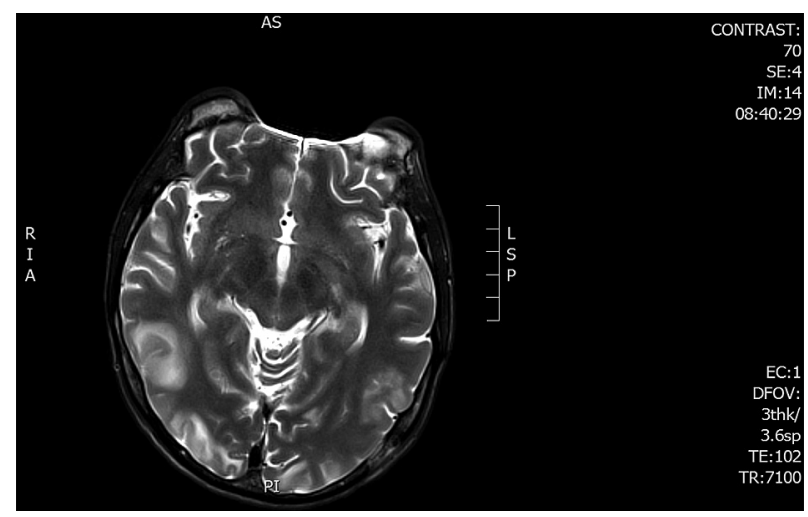

Figure 4. Magnetic resonance of the head: multiple contrast enhancing foci

noted. Foci were found in the right parietal lobe, left frontal lobe, left parietal and temporal lobes, and left hemisphere of cerebellum. The diameter of changes were from 1.5 to $5.5 \mathrm{~mm}$. Because of the small diameter of changes, differential between TB and neurotoxoplasmosis was uncertain, and after the result of the scan was obtained oral pyrimeth amine (50 mg qd) combined with clindamycin (600 mg tid) were included as empirical treatment of neurotoxoplasmosis. To establish the diagnosis further, lumbar puncture was performed: direct AFB smear was negative; however, AFB cultures were. Following the introduction of the trimethoprim/sulfamethoxazole and pyrimethamine treatment neutropenia was noted with decrease of the total leukocyte count to $0.79 \times 10^{3} / \mathrm{mm}^{3}$, neutrophils $0.0 \times 10^{3} / \mathrm{mm}^{3}$ ).

The patient remained on the first line of tuberculostatics for four months until obtaining drug-susceptibility for Mycobacterium tuberculosis (August 2011).

In the susceptibility culture, mycobacteria were resistant to the streptomycin, isoniazid, rifampicin, and ethambutol as well as erythromycin and rifabutin. Subsequently, therapy was optimised to second line of tuberculostatics: clofazimine (100 mg bid orally), levofloxacin (500 mg bid), ethionamide

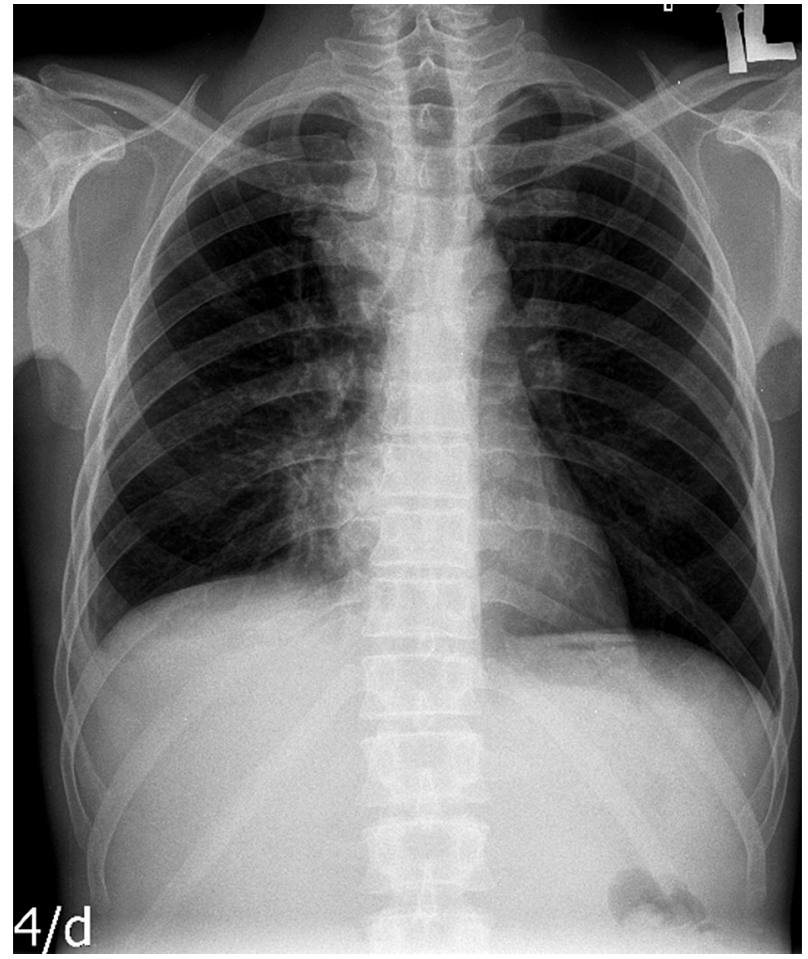

Figure 5. Chest X-ray after 6 months of the therapy

(750 mg bid orally), and clarithromycin (500 mg bid orally). After two months of new modified therapy the patient's condition significantly improved, so a new chest X-ray was taken (Figure 5).

On the chest X-ray there were inflammatory changes in middle lobe, and enlargement in the upper mediastinum, which required differential diagnostics between tumour and enlarged lymph nodes.

Follow-up lung computed tomography was performed to confirm that changes in the lungs were caused by TB. In the radiograms, uncountable nodular lesions spread throughout the lungs with divergent structure and density were found. Additionally, in the fifth segment of the right lung a tumour of $1.3 \times 0.7 \mathrm{~cm}$ diameter, in connection with pleura, was noted. Diagnosis was uncertain, with possibility of TB, fungal infection, lymphoma, or metastasis to be considered. Antituberculostatics were continued due to the high probability of existing tuberculomas. Close observation was ordered to conclude diagnosis.

Furthermore, in the head MRI in 06.2011 (Figure 6), inflammatory changes in the mastoid process were described, which caused patient's conductive hearing loss (possibly also partially caused by the streptomycin use).

Also, as in 04.2012 , the patient reported pain, swelling, and redness in the front of the right axillary region, CT of this area was performed (Figure 7): which showed enlarged subclavian bursa filled with purulent substance, so an operation was performed (AFB positive culture was obtained). 


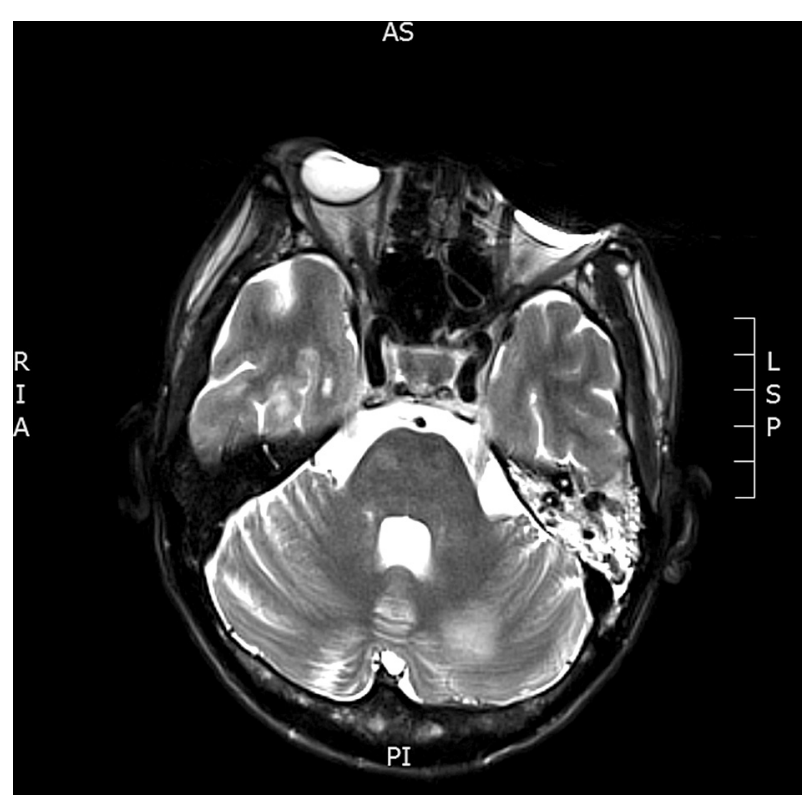

Figure 6. MRI of the brain after 1 month of therapy: inflammatory changes in the mastoid process

In 10.2012 the patient was admitted due to clinical symptoms of stroke, with right hemiparesis and motor aphasia in CT performed, a hypodense area was shown $(22 \mathrm{HU})$ in the left temporal area, suggesting ischaemic changes.

In 11.2012 surgery was performed to remove lymph nodes of upper mediastinum (from removed material, AFB cultures were found positive).

Because of persistent treatment with levofloxacin the patient suffered from recurrent (eight) inflammations of urinary tract infections caused by extended-spectrum $\beta$-lactamases Enterobacteriaceae. Treatment with carbapenems (meropenem $2000 \mathrm{mg}$ per day intravenously or ertapenem $1000 \mathrm{mg}$ per day intravenously) for minimum seven days each time followed by the intermittent fosfomycin was implemented. Carbapenem treatment was complicated by neutro- and lymphopaenia - for this reason subcutaneous G-CSF - $3 \mathrm{mln}$ IU was given daily. Recurrent urinary tract infections were complicated by post-inflammatory phimosis with necessity for surgical intervention in 2015. Antibiotic use resulted in further complications: toxic, cholestatic hepatitis 2011, reactive pancreatitis in 2012, as well as diarrhoea caused by Clostridium difficile infection in 2012. Besides that, because of the patient's serious immunodeficiency, he had in 05.2012 an HBV reactivation (HbsAg positive, HbeAg negative, anti-Hbe positive, anti-HBc-IgM negative, HBV-DNA 14778 IU/ml; elevated liver enzymes: AST 362 U/l, ALT 578 U/ml; ALP 385 U/l; GGTP 286 U/l with bilirubin level $7.0 \mathrm{mg} / \mathrm{dl}$ ) and in 03.2013 a flu infection (AH1N1) treated successfully with oseltamivir ( 2 x $75 \mathrm{mg}$ orally).

In the time of observation antiretroviral treatment was modified three times because of side effects such as neuropsychiatric abnormalities, diarrhoea, nausea, and muscular pain. At first it was changed to abacavir + lamivudine (Kivexa - qd) with lopinavir and ritonavir (Kaletra - two tablets bid), after

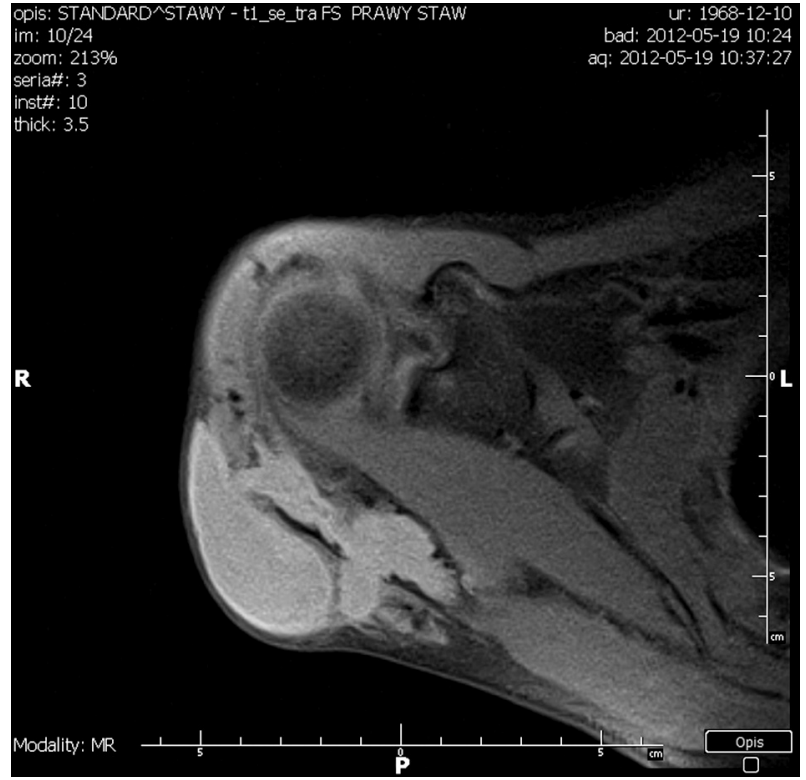

Figure 7. Computed tomography scan of the right axillary region: enlarged subclavian bursa

that in December 2011 to raltegravir (Isentress $2 \times 400 \mathrm{mg}$ daily), abacavir, and lamivudine (Kivexa qd), and finally to raltegravir (Isentress $400 \mathrm{bid}$ ) and darunavir (Prezista $600 \mathrm{mg}$ bid) boosted with ritonavir (100 mg bid). Lamivudine was withdrawn in March 2012 after seven months of treatment.

In total, during 36 months of anti-tuberculosis therapy, the patient required multiple X-rays, CTs, and MRI-scans. Radiological tests indicated multiple lesions caused by MDR-TB infection, not only in the lungs but also in liver, spleen, brain, and musculoskeletal system, and supraclavicular lesions required surgical punctuate and drainage.

Although negative sputum AFB tests after only four and five months were received, anti-TBC therapy was prolonged to 36 months due to AFB-positive cultures in punctuates or surgically removed lymph nodes. Finally, in 2014 the patient finished his TB treatment. All events occurred during the treatment process are shown in Figure 8.

Currently, the patient remains on the stable cART treatment and his latest laboratory tests (2015-2016) indicate full HIV suppression (shown HIV-RNA level $<40$ copies/ml) with partial immune reconstitution (last lymphocyte T-CD4 cells 351 cells $/ \mathrm{mm}^{3}$ ). No new opportunistic infections developed in the last year (2016). He also had no new opportunistic infections so far. Hemiparesis and motor aphasia improved in the course of physiotherapy but remains slightly noticeable.

\section{Discussion}

As for HIV and TB coinfection, special consideration of poor cell-mediated immunity should be taken into account, which often leads to multiorgan TB at the start of a treatment process [9]. Even patients with no pulmonary 
symptoms of TB or only TB in their history may often present with central nervous system (CNS) or bone TB as a first or main manifestation of the sickness [10].

Reviewing this clinical case, the background of patient nationality and country of work must be considered. It is evident, that contact with co-workers and cohabitants in Norway might have been associated with MDR-TBC acquisition. According to WHO report [4] in 2013 there were 47 (MDR-TB) from 7,250 (0.65\%) newly-diagnosed TB cases in Poland. In comparison, Norway which has one of the lowest overall $\mathrm{TB}$ infection rates, had six cases of MDR-TB among 401 (1.5\%) TB cases. Eastern Europe, however, has a very high rate of newly detected TB as well as MDR-TB - 10,585 new MDR-TB cases among 48,134 (22\%) in Ukraine and 13,521 new MDR-TB cases among 142,533 new TB cases (9.5\%) in Russia. According to this data, it is clear that because of migration even countries with relatively low risk of mycobacterial resistance may face that problem in treatment. It all shows that even countries with low levels of drug resistance may not be excluded from risk, and because of migration, more patients in Western Europe may face MDR and XDR resistance imported from Eastern parts of the continent.

Extrapulmonary and multiorgan TBC often spread to lymph nodes, gastrointestinal tract, bones, CNS, or genitourinary tract. In all these organs and tissues permanent changes in organ structures, such as bone erosion or enlarged lymph nodes, may be observed [11]. It is also important to note that stroke is one of the frequent complications of when TB process involves the central nervous system $[12,13]$.

In many similar cases [14-16] and cohort studies a surgical approach is favourable when TB, especially drug-resistant, involves regions and organs with poor drug penetrations, such as pleurae, bursas, or lymph nodes. Many of these interventions were thoracoplasty with lung or lobe excision. Studies show that a combined surgical approach and optimal drug combination may ultimately help to control the mycobacterial infection [17]. Especially in MDR or XDR TBC it is commonly necessary to carry out long, toxic treatment with limited numbers of drugs, often with poor penetration to regions with lower blood flow. Surgical punctate or excision may shorten the time of treatment and reduce the number of complications as well as side effects.

\section{Conclusions}

Patients may "import" TB from another country with higher prevalence of drug resistant strains. It should be especially considered in individuals with a history of stay in the Eastern Europe or Central Asia, but it is also possible to "import" it from countries with very low risk of infection (for example Norway). Therefore, not only the country of index case origin but also other contacts, such as co-workers and cohabitants must be considered. Multiorgan TB requires a multidisciplinary approach to the treatment, not only drug administration based on drug-susceptibility, but also surgi- cal removal of TB abscess or other infected organs or parts may be necessary.

Also, it should not be forgotten that stroke is a frequent complication when the TB process involves the CNS, and that a long-term broad-spectrum antibiotic treatment selection of bacterial strains complicating this treatment is common (e.g. genital urinary tract infections or Clostridium difficile diarrhoeas).

\section{Conflict of interest}

The author's declared no potential conflicts of interest with respect to the research, authorship, and/or publication of this article.

\section{References}

1. Daniel TM. The history of tuberculosis. Respir Med 2006; 100: 1862-1870.

2. Perelman MI, Strelzov VP. Surgery for pulmonary tuberculosis. World J Surg 1997; 21: 457-467.

3. Treatement of tuberculosis guidlines, WHO, $4^{\text {th }}$ ed. 2009. Available at: http://www.who.int/tb/publications/2010/9789241547833/en/.

4. World Health Organisation. Global tuberculosis report 2013. WHO Library Cataloguing-in-Publication Data.

5. Guidelines on co-trimoxazole prophylaxis for HIV-related infections among children, adolescents and adults in resource-limited settings: recommendations for a public health approach. World Health Organization, Geneva 2006.

6. Harries AD, Zachariah R, Lawn SD. Providing HIV care for co-infected tuberculosis patients: a perspective from sub-Saharan Africa. Int J Tuberc Lung Dis 2009; 13: 6-16.

7. Omura S, Nakaya M, Mori A, et al. M. A clinical review of 38 cases of cervical tuberculous lymphadenitis in Japan - the role of neck dissection. Auris Nasus Larynx 2016; 43: 672-676.

8. Guidelines for the programmatic management of drug-resistant tuberculosis: emergency update 2008. World Health Organization, Geneva 2008 (WHO/HTM/TB/2008.402).

9. Shenoi SV, Brooks RP, Barbour R, et al. Survival from XDR-TB is associated with modifiable clinical characteristics in rural South Africa. PLoS One 2012; 7: e31786.

10. Garg RK, Somvanshi DS. Spinal tuberculosis: A review. J Spinal Cord Med 2011; 34: 440-454.

11. Kienzl-Palma D, Prosch H. Extrathoracic manifestations of tuberculosis. Radiologe 2016; 56: 885-889.

12. Singer EJ, Valdes-Sueiras M, Commins DL, et al. HIV stroke risk: evidence and implications. Ther Adv Chronic Dis 2013; 4: 61-70.

13. Hegde SS, Ismail M, Rama K, et al. Recurrent Stroke as the First Manifestation in a Patient Infected with HIV - A Case Report. J AIDS Clin Res 2013; 4: 202.

14. Kempker RR, Vashakidze S, Solomonia N, et al. Surgical treatment of drug-resistant tuberculosis. Lancet Infect Dis 2012; 12: 157-166.

15. Chalya PL, Mchembe MD, Mshana SE, et al. Clinicopathological profile and surgical treatment of abdominal tuberculosis: a single centre experience in northwestern Tanzania. BMC Infect Dis 2013; 13: 270 .

16. Vashakidze S, Gogishvili S, Nikolaishvili K, et al. Favorable Outcomes for Multi- and Extensively Drug Resistant Tuberculosis Patients Undergoing Surgery. Ann Thorac Surg 2013; 95: 1892-1898.

17. Dheda K, Gumbo T, Maartens G, et al. The epidemiology, pathogenesis, transmission, diagnosis, and management of multidrug-resistant, extensively drug-resistant, and incurable tuberculosis. Lancet Respir Med 2017; 5: 291-360. 